\title{
Sex and age differences in isolated traumatic brain injury: a retrospective observational study
}

\author{
Sanae Hosomi ${ }^{1} 2^{*}$, Tetsuhisa Kitamura ${ }^{2}$, Tomotaka Sobue ${ }^{2}$, Hiroshi Ogura ${ }^{1}$ and Takeshi Shimazu ${ }^{1}$
}

\begin{abstract}
Background: Among the many factors that may influence traumatic brain injury (TBI) progression, sex is one of the most controversial. The objective of this study was to investigate sex differences in TBI-associated morbidity and mortality using data from the largest trauma registry in Japan.

Methods: This retrospective, population-based observational study included patients with isolated TBI, who were registered in a nationwide database between 2004 and 2018. We excluded patients with extracranial injury (Abbreviated Injury Scale score $\geq 3$ ) and removed potential confounding factors, such as non-neurological causes of mortality. Patients were stratified by age and mortality and post-injury complications were compared between males and females.
\end{abstract}

Results: A total of 51,726 patients with isolated TBI were included (16,901 females and 34,825 males). Mortality across all ages was documented in 12.01\% (2030/16901) and 12.76\% (4445/34825) of males and females, respectively. The adjusted odds ratio (OR) of TBI mortality for males compared to females was 1.32 ( $95 \%$ confidence interval [CI], 1.22-1.42]. Males aged 10-19 years and $\geq 60$ years had a significantly higher mortality than females in the same age groups (10-19 years: adjusted OR, 1.97 [95\% Cl, 1.08-3.61]; 60-69 years: adjusted OR, 1.24 [95\% Cl, 1.02-1.50]; 70-79 years: adjusted OR, 1.20 [95\% Cl, 1.03-1.40]; 80-89 years: adjusted OR, 1.50 [95\% Cl, 1.31-1.73], and 90-99 years: adjusted $\mathrm{OR}, 1.72$ [95\% Cl, 1.28-2.32]). In terms of the incidence of post-TBI neurologic and non-neurologic complications, the crude ORs were 1.29 ( $95 \% \mathrm{Cl}, 1.19-1.39)$ and 1.14 (95\% Cl, 1.07-1.22), respectively, for males versus females. This difference was especially evident among elderly patients (neurologic complications: OR, 1.27 [95\% Cl, 1.14-1.41]; non-neurologic complications: OR, 1.29 [95\% Cl, 1.19-1.39]).

Conclusions: In a nationwide sample of patients with TBI in Japan, males had a higher mortality than females. This disparity was particularly evident among younger and older generations. Furthermore, elderly males experienced more TBI complications than females of the same age.

Keywords: Mortality, Traumatic brain injury, Sex, Epidemiology, In-hospital complications

\section{Background}

Traumatic brain injury (TBI) is a major cause of mortality and morbidity worldwide [1]. While it predominantly affects younger individuals, its incidence is increasing

*Correspondence: s-hosomi@hp-emerg.med.osaka-u.ac.jp

${ }^{1}$ Department of Traumatology and Acute Critical Medicine, Osaka

University Graduate School of Medicine, 215, Yamada-oka, Suita, Japan

Full list of author information is available at the end of the article among the 65 years and older age group, especially in developed countries [2].

While prior studies have reported a sex difference in TBI mortality, a recent review of clinical and epidemiological studies concluded that there was insufficient evidence to confirm such a disparity [3]. The results of epidemiological studies on TBI have often been limited in terms of their generalizability to larger populations, due to the large heterogeneity in their patient samples; this has been a source of conflicting results 
across studies. Certain factors may increase the risk of TBI, owing to their interactions with biological, behavioral, social, and cultural conditions before and at the time of injury [4]. For example, in developed countries, the greater risk of TBI in individuals over 65 years of age has been attributed to a high incidence of falls from low heights. In contrast, decreases in high-velocity traffic accidents, improved road conditions, improved safety features in newer vehicles, and the stronger enforcement of traffic regulations have been reported to be responsible for a lower risk of TBI in the 15- to 44-year-old age group [5]. Therefore, due to an aging demographic, there is an urgent need to fully elucidate age and sex disparities in TBI incidence and outcomes.

The primary objective of this study was to assess sex differences in mortality, following age stratification, among patients treated for TBI in trauma centers. The secondary objective was to characterize sex differences in TBI complications. While the assessment of outcomes following TBI should ideally take into account the features of the specific injury (e.g., mechanism and type of injury, post-injury complications, extracerebral trauma accompanying TBI), we examined isolated TBI in order to eliminate the potential confounding effect of the severity of other associated injuries.

\section{Methods}

\section{Study design, population, and setting}

This nationwide retrospective cohort study was conducted using the Japanese Trauma Data Bank (JTDB). We included patients with TBI who were registered in the database between January 2004 and December 2018, and transported to a JTDB-associated hospital for treatment. TBI was defined as an injury to the brain due to an external force. Patients with TBI were screened with the Abbreviated Injury Scale (AIS) code [6]; we excluded cases with AIS $\geq 3$ for all other body regions (chest, abdomen, and extremity), as polytrauma associated with TBI is known to increase the risk of mortality [7]. Furthermore, we excluded patients with a maximum head AIS score of 6 (lethal injury) or 9 (unspecified injury); cardiopulmonary arrest on hospital arrival; a requirement for inter-hospital transport [8, 9]; or missing data for variables required for the logistic regression analysis. Cardiopulmonary arrest was defined as a systolic blood pressure of $0 \mathrm{mmHg}$ and/or heart rate of $0 \mathrm{bpm}$ [10].

\section{The data registry}

The JTDB was launched in 2003 by the Japanese Association for the Surgery of Trauma (Trauma Surgery Committee) and the Japanese Association for Acute Medicine (Committee for Clinical Care Evaluation) [11], and is similar to the trauma databases in North America,
Europe, and Oceania. By 2018, a total of 272 major emergency medical institutions across Japan were registered in the JTDB database [10]. The included hospitals have service levels similar to those of level I trauma centers in the United States. Data are collected from participating institutions via the internet, and physicians and medical assistants who attend the AIS coding course are the main contributors of the inputted data [10,11]. Patient data recorded in the JTDB includes the following: age, sex, mechanism of injury, AIS code (1998 version), injury severity score, vital signs on hospital arrival, date and time series from hospital arrival to discharge, medical procedures (e.g., interventional radiology, surgery, computed tomography), complications, and the date of mortality or hospital discharge $[10,11]$. This study used the most recent data available in the JTDB registry.

\section{Study endpoints}

The primary outcome was death at hospital discharge. The secondary outcomes were post-TBI in-hospital neurologic and non-neurologic complications that were diagnosed by an in-hospital medical team.

\section{Statistical analysis}

Patient characteristics were compared between groups using the unpaired $\mathrm{t}$-test for continuous variables and the chi-square test or Fisher's exact test for categorical variables. A multiple logistic regression analysis was used to assess factors potentially associated with mortality; results were expressed as odds ratios (ORs) and 95\% confidence intervals (CIs). Potential confounders included factors that were clinically essential and previously reported to be associated with variables in the multivariable analyses $[6,12,13]$. The multivariable logistic regression model for mortality due to isolated TBI was adjusted for the following variables: age (10-year strata); year of TBI onset (2004-2006, 2007-2009, 2010-2012, 20132015, 2016-2018); mechanism of trauma (motor vehicle driver, motor vehicle passenger, back seat passenger, motorcycle driver, motorcycle passenger, bicycle, pedestrian, other vehicle, fall from a great height, fall down the stairs, fall on the ground level, other blunt injury, penetrating); alcohol drinking (no/yes); use of anticoagulant or antiplatelet drugs (no, yes); hypotension on admission to the emergency department (no, yes); Glasgow Coma Scale [GCS] group on arrival [3-15]; maximum head AIS [3-5]; type of TBI (diffuse brain injury, focal brain injury, uncategorized); site of TBI $(1,2, \geq 3)$; whether an operation was indicated for TBI (no, yes), and post-TBI complications (none, neurological, non-neurological). In addition, subgroup analyses were performed to identify mortality tendencies at hospital discharge in the different age groups. The OR for mortality in males versus females 
was determined for each 10-year age stratum. As per the secondary outcome, we used a univariable analysis to compare post-TBI complications in each organ system between females and males in predetermined age groups $(0-19,20-59, \geq 60)$. Detailed comparisons of the mechanism and type of TBI by age group were also performed.

Statistical significance was defined as a two-sided p-value of less than 0.05, or assessed using a 95\% CI. All analyses were performed using STATA version 16 (StataCorp). This study was reported in accordance with the Strengthening the Reporting of Observational Studies in Epidemiology statement [14].

\section{Results}

A total of 51,726 patients with isolated TBI were included in the analysis. Among these patients, 16,901 (32.67\%) were females and 34,825 (67.33\%) were males. Figure 1 and Additional file 1 depict the flow of patients in the study and the distribution of isolated TBI cases across all ages, respectively. Patient characteristics are summarized in Table 1 . The median (interquartile range) age was higher in females (72 (54-82) years) compared to males (64 (42-77) years) (Table 1).

Blunt trauma was the most common type of injury among all patients. Falls on the ground level $(35.1 \%$ [526/16901] and 29.9\% [10397/34825] in females and males, respectively) were the most frequent cause of trauma, and the most common type of TBI was diffuse brain injury $(53.8 \%$ [9091/16901] and $54.1 \%$ [18839/34825] in females and males, respectively). The proportion of severe cases (based on GCS scores and AIS scores on admission to the emergency department) was significantly lower in females compared to males (20.6\% [3473/16901] versus $22.9 \%$ [7992/34825] for GCS, $21.8 \%$ [3691/16901] versus $24.2 \%$ [8445/34825] for AIS). Compared to males, a lower proportion of females were intoxicated with alcohol at the time of the trauma $(5.8 \%$ [979/16901] versus 20.0\% [6978/34825]; p <0.001). A greater proportion of males compared to females had multiple sites of intracranial injury (23.2\% [3926/34825] versus $25.7 \%$ [8937/16901] for two injury sites, and $11.9 \%$ [2013/34825] versus $15.9 \%$ [5551/16901] for three or more injury sites) and elevated intracranial pressure (ICP) requiring operative treatment $(17.0 \%$ [2872/34825] versus $18.4 \%$ [6406/16901]).

The risk of mortality (adjusted OR, 1.32 [95\% CI, 1.221.42] was higher for males $(12.76 \%$ [4445/34825]) than females (12.01\% [2030/16901]) across all ages, even after adjusting for confounders including age, injury severity, and medications (Fig. 2, Additional file 2). Sex differences in mortality risk in each 10-year age stratum are also shown in Fig. 2 and Additional file 2. A significant

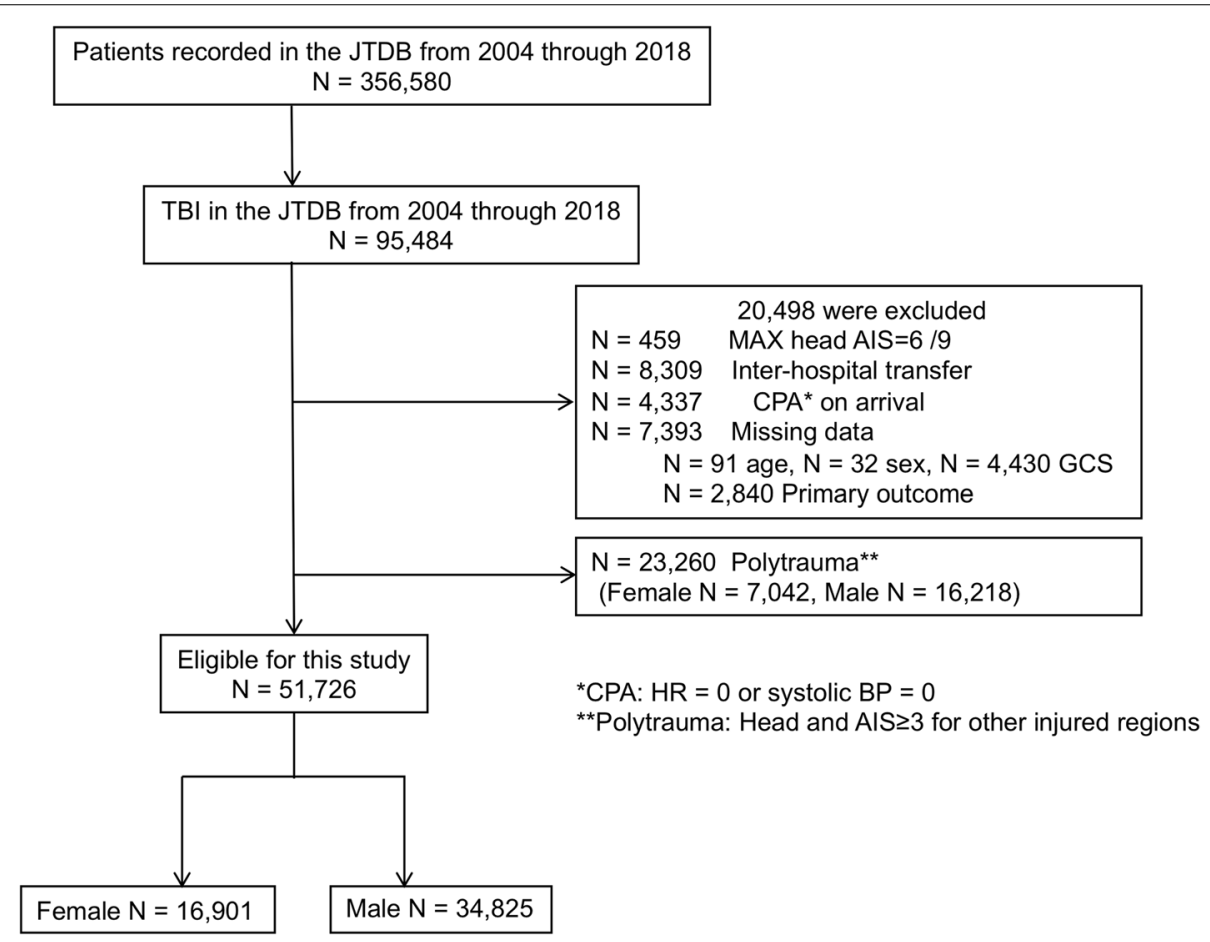

Fig. 1 Flow of patients in the study. Of the 51,726 patients included for isolated TBI, there were 34,825 (67.3\%) men and 16,901 (32.7\%) women. Of the 23,260 patients excluded for polytrauma, there were 16,218 (69.7\%) men and 7,042 (30.3\%) women. JTDB, Japan Trauma Data Bank; AIS, Abbreviated Injury Scale; CPA, cardiopulmonary arrest; GCS, Glasgow Coma Scale; MAX, maximum; TBI, traumatic brain injury 
Table 1 Comparison of baseline characteristics between males and females with isolated TBI

\begin{tabular}{|c|c|c|c|c|c|}
\hline & & $\begin{array}{l}\text { Total } \\
N=51,726\end{array}$ & $\begin{array}{l}\text { Female } \\
N=16,901\end{array}$ & $\begin{array}{l}\text { Male } \\
N=34,825\end{array}$ & $p$-value \\
\hline Age, year & median (IQR) & $67(45-79)$ & $72(54-82)$ & $64(42-77)$ & $<0.001$ \\
\hline \multicolumn{6}{|l|}{ Year of onset } \\
\hline 2004-2006 & n (\%) & 1,904 ( 3.7\%) & $613(3.6 \%)$ & 1,291 ( 3.7\%) & 0.004 \\
\hline $2007-2009$ & n (\%) & $5,990(11.6 \%)$ & $1,855(11.0 \%)$ & $4,135(11.9 \%)$ & \\
\hline 2010-2012 & n (\%) & $11,576(22.4 \%)$ & $3,743(22.1 \%)$ & $7,833(22.5 \%)$ & \\
\hline 2013-2015 & n (\%) & $17,045(33.0 \%)$ & $5,571(33.0 \%)$ & $11,474(32.9 \%)$ & \\
\hline 2016-2018 & n (\%) & $15,211(29.4 \%)$ & $5,119(30.3 \%)$ & $10,092(29.0 \%)$ & \\
\hline \multicolumn{6}{|l|}{ Cause of trauma } \\
\hline Motor vehicle driver & n (\%) & $1,822(3.5 \%)$ & $445(2.6 \%)$ & $1,377(4.0 \%)$ & $<0.001$ \\
\hline Motor vehicle passenger & n (\%) & $313(0.6 \%)$ & $156(0.9 \%)$ & $157(0.5 \%)$ & \\
\hline Back seat passenger & n (\%) & 455 ( 0.9\%) & $241(1.4 \%)$ & $214(0.6 \%)$ & \\
\hline Motorcycle driver & n (\%) & $3,834(7.4 \%)$ & $793(4.7 \%)$ & $3,041(8.7 \%)$ & \\
\hline Motorcycle passenger & n (\%) & 194 ( 0.4\%) & $80(0.5 \%)$ & $114(0.3 \%)$ & \\
\hline Bicycle & n (\%) & $6,539(12.6 \%)$ & $2,650(15.7 \%)$ & $3,889(11.2 \%)$ & \\
\hline Pedestrian & n (\%) & $5,194(10.0 \%)$ & $2,365(14.0 \%)$ & $2,829(8.1 \%)$ & \\
\hline Other vehicle & n (\%) & $225(0.4 \%)$ & 49 ( 0.3\%) & $176(0.5 \%)$ & \\
\hline Fall from a great height & n (\%) & $3,067(5.9 \%)$ & 497 ( 2.9\%) & $2,570(7.4 \%)$ & \\
\hline Fall down the stairs & n (\%) & $8,621(16.7 \%)$ & $2,451(14.5 \%)$ & $6,170(17.7 \%)$ & \\
\hline Fall on the ground level & n (\%) & $16,323(31.6 \%)$ & $5,926(35.1 \%)$ & $10,397(29.9 \%)$ & \\
\hline Other blunt injury & n (\%) & 5,086 ( 9.8\%) & $1,235(7.3 \%)$ & $3,851(11.1 \%)$ & \\
\hline Penetrating & n (\%) & $53(0.1 \%)$ & $13(0.1 \%)$ & $40(0.1 \%)$ & \\
\hline Alcohol drunk & n (\%) & $7,957(15.4 \%)$ & $979(5.8 \%)$ & $6,978(20.0 \%)$ & $<0.001$ \\
\hline Anticoagulant / antiplatelet & n (\%) & $1,509$ ( $2.9 \%)$ & 495 ( 2.9\%) & $1,014(2.9 \%)$ & 0.91 \\
\hline Hypotension (BP $\leq 90)$ on arrival & $\mathrm{n}(\%)$ & $1,395(2.7 \%)$ & 495 ( $2.9 \%)$ & $900(2.6 \%)$ & 0.023 \\
\hline GCS score on arrival & median (IQR) & $14(9-15)$ & $14(10-15)$ & $14(9-15)$ & $<0.001$ \\
\hline Severe (3-8) & $\mathrm{n}(\%)$ & $11,466(22.2 \%)$ & $3,474(20.6 \%)$ & $7,992(22.9 \%)$ & $<0.001$ \\
\hline Moderate (9-12) & n (\%) & $6,989(13.5 \%)$ & $2,078(12.3 \%)$ & $4,911(14.1 \%)$ & \\
\hline Mild (13-15) & n (\%) & $33,271(64.3 \%)$ & $11,349(67.1 \%)$ & $21,922(62.9 \%)$ & \\
\hline \multicolumn{6}{|l|}{ Type of TBI } \\
\hline Diffuse brain injury & n (\%) & $27,930(54.0 \%)$ & $9,091(53.8 \%)$ & $18,839(54.1 \%)$ & 0.36 \\
\hline Focal brain injury & $n(\%)$ & $20,008(38.7 \%)$ & $6,533(38.7 \%)$ & $13,475(38.7 \%)$ & \\
\hline Uncategorized & n (\%) & $3,788(7.3 \%)$ & $1,277(7.6 \%)$ & $2,511(7.2 \%)$ & \\
\hline Injury site & median (IQR) & $1(1-2)$ & $1(1-2)$ & $1(1-2)$ & $<0.001$ \\
\hline 1 & n (\%) & $31,299(60.5 \%)$ & $10,962(64.9 \%)$ & $20,337(58.4 \%)$ & $<0.001$ \\
\hline 2 & n (\%) & $12,863(24.9 \%)$ & $3,926(23.2 \%)$ & $8,937(25.7 \%)$ & \\
\hline$\geq 3$ & n (\%) & $7,564(14.6 \%)$ & $2,013(11.9 \%)$ & $5,551(15.9 \%)$ & \\
\hline Max head AIS & median (IQR) & $4(3-4)$ & $4(3-4)$ & $4(3-4)$ & $<0.001$ \\
\hline 3 & $\mathrm{n}(\%)$ & $17,355(33.6 \%)$ & $5,864(34.7 \%)$ & $11,491(33.0 \%)$ & $<0.001$ \\
\hline 4 & n (\%) & $22,235(43.0 \%)$ & $7,346(43.5 \%)$ & $14,889(42.8 \%)$ & \\
\hline 5 & n (\%) & $12,136(23.5 \%)$ & $3,691(21.8 \%)$ & 8,445 (24.2\%) & \\
\hline Operation for elevated ICP & n (\%) & $9,278(17.9 \%)$ & $2,872(17.0 \%)$ & $6,406(18.4 \%)$ & $<0.001$ \\
\hline \multicolumn{6}{|l|}{ Complication } \\
\hline None & n (\%) & $44,035(85.1 \%)$ & $14,662(86.8 \%)$ & $29,373(84.3 \%)$ & $<0.001$ \\
\hline Neurological & n (\%) & 3,095 ( 6.0\%) & 857 ( 5.1\%) & 2,238 ( 6.4\%) & \\
\hline Non-neurological & n (\%) & 4,596 ( 8.9\%) & $1,382(8.2 \%)$ & $3,214(9.2 \%)$ & \\
\hline
\end{tabular}

$T B I$ traumatic brain injury, GCS Glasgow Coma Scale, BP blood pressure, AIS abbreviated injury scale, ICP intracranial pressure, IQR interquartile range, max maximum 


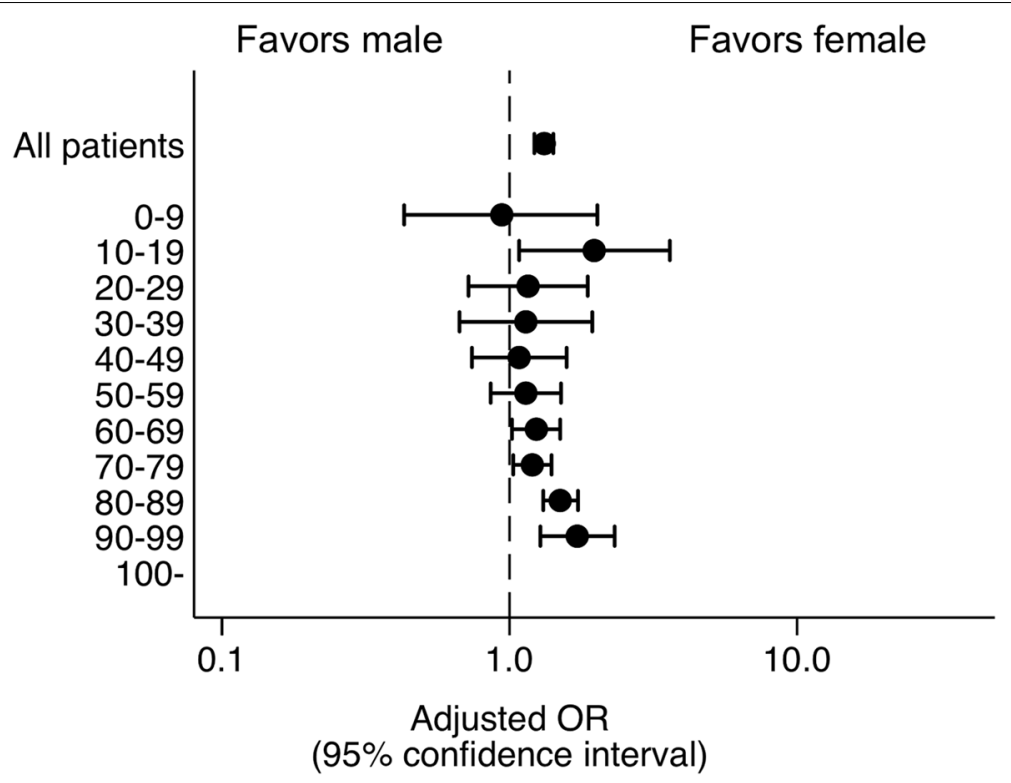

Fig. 2 Mortality risk in males and females with traumatic brain injury within 10-year age strata. Adjusted OR for death at hospital discharge is shown. OR, odds ratio

disparity was observed in the following age groups: 10-19 years (adjusted OR, 1.97 [95\% CI, 1.08-3.61]), 60-69 years (adjusted OR, 1.24 [95\% CI, 1.02-1.50]), 70-79 years (adjusted OR, 1.20 [95\% CI, 1.03-1.40]), 80-89 years (adjusted OR, 1.50 [95\% CI, 1.31-1.73]), and 90-99 years (adjusted OR, 1.72 [95\% CI, 1.28-2.32]). Among the mechanisms of injury, back seat passenger (adjusted OR $=2.6595 \% \mathrm{CI}: 1.65-4.28$ ) and fall from a greater height (adjusted OR $=2.82$ 95\% CI: 2.15-3.69) were especially attributed to mortality compared to motor vehicle driver (Table 2). Table 2 also shows that neurological complication showed less effect on mortality (adjusted OR $=0.40$ 95\% CI: $0.34-0.47$ ) while nonneurological complication showed more effect (adjusted $\mathrm{OR}=1.66$, 95\% CI: $1.52-1.82$ ).

Table 3 shows that males had an increased risk of neurological complications (e.g., diabetes insipidus, hydrocephalus, cerebrospinal fluid fistula, meningitis, post traumatic stress syndrome, cognitive dysfunction) (crude OR, 1.29 [95\% CI, 1.19-1.39] and non-neurological complications (crude OR, 1.14 [95\% CI, 1.07-1.22]); this was particularly evident among patients 60 years of age and over (crude OR, 1.27 [95\% CI, 1.14-1.41] for neurological complications; crude OR, 1.29 [95\% CI, 1.19-1.39] for non-neurological complications). Males in this age group also exhibited greater risks for respiratory complications (e.g., acute respiratory distress syndrome [ARDS]) (crude OR, 1.28 [95\% CI, 1.03-1.59]), digestive complications (e.g., gastrointestinal bleeding) (crude OR, 1.34 [95\% CI, 1.08-1.66]), urinary complications (e.g., acute renal dysfunction) (crude OR, 2.02 [95\% CI, 1.16-3.53]), and infections (e.g., acute pneumonia, other organ infections) (crude OR, 1.47 [95\% CI, 1.33-1.62]) (Table 3).

Differences in the cause and type of TBI between males and females were more evident in older age groups (Additional file 3). The proportion of TBI cases attributed to injuries sustained in traffic accidents involving back seat passenger, bicycles or pedestrians was significantly greater among females compared to males, across all age groups. While falls from a great height were significantly more often cited as the cause of TBI among adult males (crude OR, 1.66 [95\% CI, 1.41-1.96] for the 20-59-year-old age group; crude OR, 4.95 [95\% CI, 4.215.85 ] for the $\geq 60$-year-old age group), TBI due to falls at the ground level were more common in elderly females (crude OR, 0.87 [95\% CI, 0.83-0.91]). Subarachnoid hemorrhage was the leading type of TBI in both males and females, across all age groups. Focal injuries, including contusion, epidural hemorrhage, intracranial hemorrhage, and diffuse axonal injury were more common in elderly males; an exception was acute subdural hematoma, which was more frequently documented in elderly females compared to elderly males (crude OR, 0.93 [95\% CI, 0.89-0.97]).

\section{Discussion}

The results of this study indicated that sex differences in TBI-associated mortality and complications were affected by patient age. While such mortality was higher in males than females, this disparity was particularly 
Table 2 Multivariable analysis of factors associated with the primary outcomes

\begin{tabular}{|c|c|c|}
\hline & Adjusted OR & $95 \% \mathrm{Cl}$ \\
\hline Gender & 1.32 & $(1.22-1.42)$ \\
\hline Age (for 10-year increment) & 1.36 & $(1.34-1.39)$ \\
\hline Year of onset (for 3-year increment) & 0.82 & $(0.80-0.85)$ \\
\hline \multicolumn{3}{|l|}{ Mechanism of injury } \\
\hline Motor vehicle driver & (reference) & \\
\hline Motor vehicle passenger & 1.73 & $(0.98-3.05)$ \\
\hline Back seat passenger & 2.65 & $(1.65-4.28)$ \\
\hline Motorcycle Driver & 1.18 & $(0.89-1.56)$ \\
\hline Motorcycle Passenger & 1.52 & $(0.74-3.13)$ \\
\hline Bicycle & 1.85 & $(1.43-2.39)$ \\
\hline Pedestrian & 2.21 & $(1.71-2.86)$ \\
\hline Other vehicle & 1.97 & $(1.12-3.44)$ \\
\hline Fall from a great height & 2.82 & $(2.15-3.69)$ \\
\hline Fall down the stairs & 1.82 & $(1.41-2.34)$ \\
\hline Fall on the ground level & 1.85 & $(1.45-2.37)$ \\
\hline Other blunt injury & 1.89 & $(1.47-2.45)$ \\
\hline Penetrating & 2.90 & $(1.04-8.13)$ \\
\hline Alcohol drunk & 0.51 & $(0.46-0.57)$ \\
\hline Anticoagulant / antiplatelet & 1.51 & $(1.26-1.80)$ \\
\hline Hypotension ( $\mathrm{BP} \leq 90$ ) on arrival & 2.70 & $(2.29-3.18)$ \\
\hline $\begin{array}{l}\text { GCS score on arrival (for 1-score incre- } \\
\text { ment) }\end{array}$ & 0.72 & $(0.72-0.73)$ \\
\hline \multicolumn{3}{|l|}{ Type of TBI } \\
\hline Diffuse brain injury & (reference) & \\
\hline Focal brain injury & 0.90 & $(0.82-0.99)$ \\
\hline Uncategorized & 0.84 & $(0.74-0.96)$ \\
\hline \multicolumn{3}{|l|}{ Injury site (n) } \\
\hline 1 & (reference) & \\
\hline 2 & 1.11 & $(1.01-1.23)$ \\
\hline $3 \leq$ & 1.11 & $(0.99-1.25)$ \\
\hline \multicolumn{3}{|l|}{ Max head AIS } \\
\hline 3 & (reference) & \\
\hline 4 & 2.39 & $(2.09-2.73)$ \\
\hline 5 & 7.50 & $(6.58-8.54)$ \\
\hline Operation for elevated ICP & 0.69 & $(0.64-0.74)$ \\
\hline \multicolumn{3}{|l|}{ Complication } \\
\hline None & (reference) & \\
\hline Neurological & 0.40 & $(0.34-0.47)$ \\
\hline Non-neurological & 1.66 & $(1.52-1.82)$ \\
\hline
\end{tabular}

$T B I$ traumatic brain injury, GCS Glasgow Coma Scale, BP blood pressure, AIS abbreviated injury scale, ICP intracranial pressure, IQR interquartile range, max maximum, $O R$ odds ratio, $\mathrm{Cl}$ confidence interval

evident among younger and older age groups. Furthermore, elderly males with TBI experienced more neurological and non-neurological complications than females of the same age.

Our finding that males had a higher mortality rate after TBI compared to females of the same age is supported by prior studies utilizing large samples $(>10,000$ patients in multiple trauma centers) obtained from national registries [3]. However, some studies with smaller sample sizes have reported that females have worse clinical outcomes than males after TBI [15-17]. Gupte et al. suggested that these contrasting results could be attributed to TBI heterogeneity [3], as well as differences in TBI severity and patient age, race, and physical condition. In addition, the majority of studies which have investigated sex differences in TBI-associated mortality have included cases with injuries to other body regions $[6,18-20]$. In order to remove this potentially confounding effect, we only included patients with isolated TBI. Furthermore, our study was restricted to patients in Japan; this reduced the confounding effects of race and injury type (the majority of TBI cases in Japan are attributed to blunt injuries).

Our study showed sex and age differences in TBI characteristics. TBI is typically classified according to clinical severity, with severe injuries usually categorized on the basis of a total GCS score of 8 or less, or anatomical severity based on the AIS score $(3=$ serious, $4=$ severe, $5=$ critical) $[3,6,8]$. In the present study, injury severity (in terms of both anatomical and physiological scales) was higher in males than females. This may be attributed to the greater prevalence of high-impact trauma and alcohol intoxication among males. Previous studies have reported that falls are the most common cause of TBI among older adults $[21,22]$. Nevertheless, we found that TBI due to falls at the ground level were more common in elderly females compared to elderly males, while falls from greater heights were more frequently documented among elderly males (Additional file 2). Furthermore, fall from a great height was the most significant risk factor for mortality among blunt trauma (Table 2). Although we found that alcohol intake was associated with the lower mortality (adjusted OR $=0.51$ ) in this study, the definitive mechanism was unclear. Alcohol contributes substantially to the morbidity of trauma patients, regardless of the type of injury suffered [23]. The results of this study suggest that males are more prone to severe injuries due to high-impact trauma; this may be due in large part to the effects of age and alcohol intoxication on defensive ability and alertness.

Injury type also affects mortality. Brain contusions and intracerebral, subdural, and fast-growing non-evacuated epidural hematomas pose a high risk of increased ICP and subsequent severe disability or mortality. In contrast, the risk of an increase in ICP is low for axonal injury, traumatic subarachnoid hemorrhage, and petechial bleeding $[24,25]$. Subdural hematomas are typically associated with more severe types of TBI and are more frequently diagnosed in female patients [26]. Indeed, it has been reported that females are significantly more likely to 
Table 3 Comparison of TBI complications between males and females in each age group

\begin{tabular}{|c|c|c|c|c|c|}
\hline & Total & Female & Male & Crude OR & $(95 \% \mathrm{Cl})$ \\
\hline All patients & $N=51,726$ & $N=16,901$ & $N=34,825$ & & \\
\hline Neurological complication & $3,095(6.0 \%)$ & $857(5.1 \%)$ & $2,238(6.4 \%)$ & 1.29 & $(1.19-1.39)$ \\
\hline Non-neurological complication & $4,596(8.9 \%)$ & $1,382(8.2 \%)$ & $3,214(9.2 \%)$ & 1.14 & $(1.07-1.22)$ \\
\hline $0-19$ & $N=5,198$ & $N=1,522$ & $N=3,676$ & & \\
\hline Neurological complication & $306(5.9 \%)$ & $86(5.7 \%)$ & $220(6.0 \%)$ & 1.06 & $(0.82-1.37)$ \\
\hline Non-neurological complication & $188(3.6 \%)$ & $64(4.2 \%)$ & $124(3.4 \%)$ & 0.80 & $(0.58-1.08)$ \\
\hline Circulatory & $26(0.5 \%)$ & $9(0.6 \%)$ & $17(0.5 \%)$ & 0.78 & $(0.35-1.76)$ \\
\hline Respiratory & $35(0.7 \%)$ & $10(0.7 \%)$ & $25(0.7 \%)$ & 1.04 & $(0.50-2.16)$ \\
\hline Digestive & $21(0.4 \%)$ & $4(0.3 \%)$ & $17(0.5 \%)$ & 1.76 & $(0.59-5.25)$ \\
\hline Urinary & $4(0.1 \%)$ & $1(0.1 \%)$ & $3(0.1 \%)$ & 1.24 & $(0.13-11.95)$ \\
\hline Coagulation & $27(0.5 \%)$ & $12(0.8 \%)$ & $15(0.4 \%)$ & 0.52 & $(0.24-1.10)$ \\
\hline Infection & $96(1.8 \%)$ & $30(2.0 \%)$ & $66(1.8 \%)$ & 0.91 & $(0.59-1.41)$ \\
\hline $20-59$ & $N=14,534$ & $N=3,412$ & $N=11,122$ & & \\
\hline Neurological complication & $1,140(7.8 \%)$ & $238(7.0 \%)$ & $902(8.1 \%)$ & 1.18 & $(1.01-1.37)$ \\
\hline Non-neurological complication & $1,033(7.1 \%)$ & $229(6.7 \%)$ & $804(7.2 \%)$ & 1.08 & $(0.93-1.26)$ \\
\hline Circulatory & $176(1.2 \%)$ & $42(1.2 \%)$ & $134(1.2 \%)$ & 0.98 & $(0.69-1.39)$ \\
\hline Respiratory & $147(1.0 \%)$ & $29(0.8 \%)$ & $118(1.1 \%)$ & 1.25 & $(0.83-1.88)$ \\
\hline Digestive & $140(1.0 \%)$ & $28(0.8 \%)$ & $112(1.0 \%)$ & 1.23 & $(0.81-1.86)$ \\
\hline Urinary & $18(0.1 \%)$ & $1(0.0 \%)$ & $17(0.2 \%)$ & 5.22 & $(0.69-39.25)$ \\
\hline Coagulation & $107(0.7 \%)$ & $20(0.6 \%)$ & $87(0.8 \%)$ & 1.34 & $(0.82-2.18)$ \\
\hline Infection & $541(3.7 \%)$ & $111(3.3 \%)$ & 430 ( $3.9 \%)$ & 1.20 & $(0.97-1.48)$ \\
\hline $60 \leq$ & $N=31,994$ & $N=11,967$ & $N=20,027$ & & \\
\hline Neurological complication & $1,649(5.2 \%)$ & $533(4.5 \%)$ & $1,116(5.6 \%)$ & 1.27 & $(1.14-1.41)$ \\
\hline Non-neurological complication & $3,375(10.5 \%)$ & $1,089(9.1 \%)$ & $2,286(11.4 \%)$ & 1.29 & $(1.19-1.39)$ \\
\hline Circulatory & $826(2.6 \%)$ & $292(2.4 \%)$ & $534(2.7 \%)$ & 1.10 & $(0.95-1.27)$ \\
\hline Respiratory & $380(1.2 \%)$ & $121(1.0 \%)$ & $259(1.3 \%)$ & 1.28 & $(1.03-1.59)$ \\
\hline Digestive & $388(1.2 \%)$ & $120(1.0 \%)$ & $268(1.3 \%)$ & 1.34 & $(1.08-1.66)$ \\
\hline Urinary & $70(0.2 \%)$ & $16(0.1 \%)$ & $54(0.3 \%)$ & 2.02 & $(1.16-3.53)$ \\
\hline Coagulation & $337(1.1 \%)$ & $139(1.2 \%)$ & $198(1.0 \%)$ & 0.85 & $(0.68-1.06)$ \\
\hline Infection & $1,927(6.0 \%)$ & $566(4.7 \%)$ & $1,361(6.8 \%)$ & 1.47 & $(1.33-1.62)$ \\
\hline
\end{tabular}

OR odds ratio, $\mathrm{Cl}$ confidence interval

experience brain swelling and intracranial hypertension than male patients with a comparable injury severity [27]. However, as the proportion of cases with multiple intracranial injury sites or elevated ICP requiring operative treatment was lower among females, we presume that the overall incidence of raised ICP was also lower compared to males.

TBI in older patients typically results from low-energy impacts such as ground-level falls, resulting in a lower proportion of subdural and epidural hematomas, as well as contusions, compared to younger patients [26]. The link between a lower incidence of raised ICP and high initial GCS scores may be explained by cerebral atrophy and an increased cerebrospinal fluid space, which may buffer new pathological intracranial masses [26]. While the JTDB did not document the deterioration of clinical symptoms, such as decreases in GCS scores by two or more points; this suggested that mortality was affected, even if the initial GCS scores on hospital admission were mild, by these factors that were not adjusted for in the present study [28]. However, as the distribution of symptom deterioration is age-dependent [28], age can significantly impact clinical outcomes by interacting with other confounding factors.

Morbidity and mortality in patients with TBI are affected by both intracranial and extracranial complications $[29,30]$. Our study reveals that older males have more neurological and non-neurological complications than females of the same age. The association of non-neurological complication with higher mortality suggested that efforts to mitigate post-trauma complications might be an important target for intervention. Indeed, these complications impact in-hospital mortality, especially in elderly patients [31]. TBI triggers a complex cascade of 
molecular and cellular events associated with a systemic inflammatory reaction and secondary extracranial organ damage [29-31]. Circulating pro-inflammatory cytokines and chemokines following TBI are potentially responsible for ARDS and acute kidney injury [32,33]. Patients with severe brain injury often require a deep level of sedation and the administration of neuromuscular blocking agents to control ICP [34]. The greater incidence of acute pneumonia may be partially explained as a side effect of this treatment strategy. It has been reported that acute lung injury and ARDS develop in $20-25 \%$ of patients with isolated brain injuries, and are associated with a threefold increased risk of mortality [35]. Furthermore, it has been suggested that ARDS is an independent predictor of mortality [36]. Mortality due to ARDS in intensive care units has decreased with recent advancements in medical technology [36]; this is also likely to reduce mortality due to TBI-associated neurological complications. Nevertheless, males tend to have more complications after TBI, as well as poorer prognoses and clinical outcomes.

The causes of sex differences in mortality following TBI remain to be determined. Preclinical studies of TBI have shown inconsistent results with regard to the neuroprotective effects of sex hormones; these include the reduction of ICP, and the improvement of cerebral perfusion pressure and neurological scores [37]. It has been reported that pubescent females have lower mortality rates after isolated moderate-to-severe TBI, compared to prepubescent females [38, 39]. Taking into account published data on the mean age of menarche $(12.2+0.9$ years $)$ and menopause $(49.5+3.5$ years $)$ among Japanese females [40], we did not observe a lower TBI-associated mortality among females of reproductive age in the present study. This is inconsistent with a prior study that reported a better prognosis in premenopausal females due to increased levels of sex hormones [15]. On the other hand, another large multicenter study found that mortality in the $>50$-year-old age group was lower in women compared to men [19]. Nevertheless, the results of clinical studies have shown that the neuroprotective mechanisms of estrogen are still controversial [41, 42]. The relative contribution of sex hormones (compared to the range of other factors identified in the present study) to sex differences in morbidity and mortality after TBI remains to be determined by future studies.

Males and females exhibit differences in terms of their developmental environment, as well as their neurodevelopment and sociological attributes; it must be acknowledged that these factors may contribute to differences in TBI incidence [43]. Our study demonstrated poorer outcomes in male TBI patients even after adjusting for various confounding factors, which may possibly be related to the intrinsic attributes of the male sex, with a greater risk of death from TBI; alternatively, these results may reflect an increased vulnerability to TBI [43]. Age was found to be an important modifier of sex differences in TBI outcomes. A better understanding of sex differences in TBI may facilitate the development of optimal treatments aimed at improving patient outcomes following TBI. The logical next step in this field of research would be to investigate sex differences in TBI cases associated with polytrauma.

\section{Limitations}

This study has several limitations. First, the use of a retrospective study design and data from a trauma registry prevented a more detailed examination of the patient population. For example, while the type of in-hospital treatment provided (e.g., hypothermia) may have affected survival after TBI, this was not documented in the JTDB. It is reported that the use of helmet was associated with greatly reduced risk of head injury in bicycle collisions with motor vehicles, and the more severe the injury considered, the greater the reduction [44]. Therefore, wearing a helmet or a seat belt is one of important factors, although JTDB did not obtain this information. Furthermore, hypoxemia in the field or in the ED and loss of pupillary reactivity, which are reported as the most important predictors of outcome in TBI in the IMPACT study [45], were unavailable in the JTDB. Moreover, we only assessed in-hospital outcomes, and did not evaluate post-trauma quality of life. Additionally, the data included in this study primarily involved cases of blunt trauma treated in trauma centers in Japan; therefore, the results cannot be generalized to other countries which have a greater proportion of penetrating injuries and non-trauma centers. Third, unmeasured confounding factors may have influenced the association between TBI and the study outcomes. For example, patient smoking habits and the deterioration of clinical symptoms may have affected the incidence of non-neurological complications and mortality, respectively. Lastly, as with all epidemiologic studies, ascertainment bias may have affected the integrity and validity of the data; nevertheless, this was minimized with the use of uniform data collection methods based on the JTDB registration system. Furthermore, the risk of potential bias was also reduced by utilizing the largest trauma database in Japan, which provided a greater statistical power for the detection of sex differences in TBI-associated mortality.

\section{Conclusions}

The results of this study suggested that males had a higher mortality rate than females after TBI. This disparity was especially evident among teenagers and those older than 60 years of age. Furthermore, elderly males experienced 
more post-TBI complications than females of the same age. The elucidation of sex differences in TBI morbidity and mortality may facilitate the development of optimal treatments aimed at improving patient outcomes.

\section{Abbreviations}

AIS: Abbreviated Injury Scale; Cl: Confidence interval; GCS: Glasgow Coma Scale; ICP: Intracranial pressure; JTDB: Japanese Trauma Data Bank; OR: Odds ratio; TBI: Traumatic brain injury.

\section{Supplementary Information}

The online version contains supplementary material available at https://doi. org/10.1186/s12883-021-02305-6.

Additional file 1. Histogram of isolated traumatic brain injury patients. Sex disparity was reversed among patients 90 years of age and over

Additional file 2. Mortality risk in males and females with traumatic brain injury. The risk of mortality was higher for males than females across all ages, even after adjusting for confounders including age, injury severity, and medications.

Additional file 3. Comparison of causes and types of traumatic brain injury between males and females in each age group. Differences in the cause and type of traumatic brain injury between males and females were more evident in older age groups

\section{Acknowledgements}

The authors thank the emergency medical service personnel, nurses, neurosurgeon, and emergency physicians who participated in the JTDB. We thank our colleagues from the Osaka University Center of Medical Data Science and Advanced Clinical Epidemiology Investigator's Research Project for providing insight and expertise for our research.

\section{Authors' contributions}

SH, T. Sobue, and TK designed the study and wrote the manuscript. SH and TK performed all statistical analyses. HO and T. Shimazu critically revised the manuscript for important intellectual content. All authors read and approved the final manuscript.

\section{Funding}

This work was supported by a Grant-in-Aid for Scientific Research (C) from the Japan Society for the Promotion of Science (18K08886) and a grant from Zenkyoren (National Mutual Insurance Federation of Agricultural Cooperatives) to SH. The funders had no role in study design, data collection and analysis, decision to publish, or preparation of the manuscript.

\section{Availability of data and materials}

The data that support the findings of this study are available from the JTDB, but restrictions apply to the availability of these data, which were used under license for the current study. Therefore, the data are not publicly available. However, the data are available from the authors upon reasonable request and with permission of the JTDB.

\section{Declarations}

\section{Ethics approval and consent to participate}

This study was approved by the ethics committee of Osaka University Graduate School of Medicine (No. 16260). Personal identifiers were removed beforehand from the JTDB; thus, the need for informed consent was waived.

\section{Consent for publication}

Not applicable.

\section{Competing interests}

The authors declare that they have no competing interests.

\section{Author details}

'Department of Traumatology and Acute Critical Medicine, Osaka University Graduate School of Medicine, 215, Yamada-oka, Suita, Japan. ${ }^{2}$ Division of Environmental Medicine and Population Sciences, Department of Social and Environmental Medicine, Osaka University Graduate School of Medicine, 215, Yamada-oka, Suita, Japan.

Received: 5 April 2021 Accepted: 22 June 2021

Published online: 05 July 2021

\section{References}

1. Roozenbeek B, Maas Al, Menon DK. Changing patterns in the epidemiology of traumatic brain injury. Nat Rev Neurol. 2013:9:231-6.

2. Majdan M, Plancikova D, Brazinova A, Rusnak M, Nieboer D, Feigin V, et al. Epidemiology of traumatic brain injuries in Europe: a cross-sectional analysis. Lancet Public Health. 2016;1:e76-83.

3. Gupte R, Brooks W, Vukas R, Pierce J, Harris J. Sex differences in traumatic brain injury: what we know and what we should know. J Neurotrauma. 2019;36:3063-91.

4. Mollayeva T, Mollayeva S, Colantonio A. Traumatic brain injury: sex, gender and intersecting vulnerabilities. Nat Rev Neurol. 2018;14:711-22.

5. Centers for Disease Control and Prevention. TBI data and statistics. http:// www.cdc.gov/traumaticbraininjury/data/; 2019. Accessed 25 Mar 2021.

6. Berry C, Ley EJ, Tillou A, Cryer G, Margulies DR, Salim A. The effect of gender on patients with moderate to severe head injuries. J Trauma. 2009:67:950-3.

7. Tachino J, Katayama Y, Kitamura T, Kiyohara K, Nakao S, Umemura Y, et al. Assessment of the interaction effect between injury regions in multiple injuries: a nationwide cohort study in Japan. J Trauma Acute Care Surg. 2021;90:185-90.

8. The abbreviated injury scale, 1990 revision, Update 98. Barrington: Association for the Advancement of Automatic Medicine; 2001.

9. Härtl R, Gerber LM, lacono L, Ni Q, Lyons K, Ghajar J. Direct transport within an organized state trauma system reduces mortality in patients with severe traumatic brain injury. J Trauma. 2006;60:1250-6.

10. Katayama Y, Kitamura T, Hirose T, Kiguchi T, Matsuyama T, Takahashi $H_{\text {, }}$ et al. Pelvic angiography is effective for emergency pediatric patients with pelvic fractures: a propensity-score-matching study with a nationwide trauma registry in Japan. Eur J Trauma Emerg Surg. 2021;47:515-f21.

11. Japan Trauma Care and Research. Japan Trauma Data Bank Annual Report 2014-2018. http://www.jast-hp.org/trauma/pdf/jtdb2019e.pdf; 2019. Accessed 25 Mar 2021.

12. Tohme S, Delhumeau C, Zuercher M, Haller G, Walder B. Prehospital risk factors of mortality and impaired consciousness after severe traumatic brain injury: an epidemiological study. Scand J Trauma Resusc Emerg Med. 2014;22:1.

13. Steyerberg EW, Mushkudiani N, Perel P, Butcher I, Lu J, McHugh GS, et al. Predicting outcome after traumatic brain injury: development and international validation of prognostic scores based on admission characteristics. PLOS Med. 2008;5:e165.

14. Gallo V, Egger M, McCormackV, Farmer PB, loannidis JP, Kirsch-Volders $M$, et al. STrengthening the Reporting of OBservational studies in Epidemiology-Molecular Epidemiology STROBE-ME: an extension of the STROBE statement. J Clin Epidemiol. 2011;64:1350-63.

15. Bayir H, Marion DW, Puccio AM, Wisniewski SR, Janesko KL, Clark RS, et al. Marked gender effect on lipid peroxidation after severe traumatic brain injury in adult patients. J Neurotrauma. 2004;21:1-8.

16. Farace $E$, Alves WM. Do women fare worse: a metaanalysis of gender differences in traumatic brain injury outcome. J Neurosurg. 2000;93:539-45.

17. Wagner AK, Sasser HC, Hammond FM, Wiercisiewski D, Alexander J. Intentional traumatic brain injury: epidemiology, risk factors, and associations with injury severity and mortality. J Trauma. 2000;49:404-10.

18. Brazinova A, Mauritz W, Leitgeb J, Wilbacher I, Majdan M, Janciak I, et al. Outcomes of patients with severe traumatic brain injury who have Glasgow Coma Scale scores of 3 or 4 and are over 65 years old. J Neurotrauma. 2010:27:1549-55.

19. Davis DP, Douglas DJ, Smith W, Sise MJ, Vilke GM, Holbrook TL, et al. Traumatic brain injury outcomes in pre- and post-menopausal females versus age-matched males. J Neurotrauma. 2006;23:140-8. 
20. Leitgeb J, Mauritz W, Brazinova A, Janciak I, Majdan M, Wilbacher I, et al. Effects of gender on outcomes after traumatic brain injury. J Trauma. 2011;71:1620-6.

21. Dams-O'Connor K, Cuthbert JP, Whyte J, Corrigan JD, Faul M, HarrisonFelix C. Traumatic brain injury among older adults at level I and II trauma centers. J Neurotrauma. 2013;30:2001-13.

22. Scheetz LJ. Injury patterns, severity and outcomes among older adults who sustained brain injury following a same level fall: a retrospective analysis. Int Emerg Nurs. 2015;23:162-7.

23. Stewart RM, Myers JG, Dent DL, Ermis P, Gray GA, Villarreal R, et al. Seven hundred fifty-three consecutive deaths in a Level I Trauma Center: the argument for injury prevention. J Trauma. 2003;54:66-70.

24. Lee TT, Galarza M, Villanueva PA. Diffuse axonal injury (DAI) is not associated with elevated intracranial pressure (ICP). Acta Neurochir. 1998;140:41-6.

25. Maas Al, Steyerberg EW, Butcher I, Dammers R, Lu J, Marmarou A, et al. Prognostic value of computerized tomography scan characteristics in traumatic brain injury: results from the IMPACT study. J Neurotrauma. 2007;24:303-14.

26. Stocchetti N, Carbonara M, Citerio G, Ercole A, Skrifvars MB, Smielewski P, et al. Severe traumatic brain injury: targeted management in the intensive care unit. Lancet Neurol. 2017;16:452-64.

27. Farin A, Deutsch R, Biegon A, Marshall LF. Sex-related differences in patients with severe head injury: greater susceptibility to brain swelling in female patients 50 years of age and younger. J Neurosurg. 2003;98:32-6.

28. Marincowitz C, Lecky FE, Townend W, Borakati A, Fabbri A, Sheldon TA. The risk of deterioration in GCS13-15 patients with traumatic brain injury identified by computed tomography imaging: a systematic review and meta-analysis. J Neurotrauma. 2018;35:703-18.

29. Zygun D. Non-neurological organ dysfunction in neurocritical care: impact on outcome and etiological considerations. Curr Opin Crit Care. 2005;11:139-43.

30. Mascia L, Sakr Y, Pasero D, Payen D, Reinhart K, Vincent JL, et al. Extracranial complications in patients with acute brain injury: a post-hoc analysis of the SOAP study. Intensive Care Med. 2008;34:720-7.

31. Adediran T, Drumheller BC, McCunn M, Stein DM, Albrecht JS. Sex differences in in-hospital complications among older adults after traumatic brain injury. J Surg Res. 2019;243:427-33.

32. Civiletti F, Assenzio B, Mazzeo AT, Medica D, Giaretta F, Deambrosis I, et al. Acute tubular injury is associated with severe traumatic brain injury: in vitro study on human tubular epithelial cells. Sci Rep. 2019;9:6090.

33. Kerr NA, de Rivero Vaccari JP, Abbassi S, Kaur H, Zambrano R, Wu S, et al. Traumatic brain injury-induced acute lung injury: evidence for activation and inhibition of a neural-respiratory-inflammasome axis. J Neurotrauma. 2018;35:2067-76
34. Carney N, Totten AM, O'Reilly C, Ullman JS, Hawryluk GW, Bell MJ, et al. Guidelines for the management of severe traumatic brain injury, fourth edition. Neurosurgery. 2017;80:6-15.

35. Holland MC, Mackersie RC, Morabito D, Campbell AR, Kivett VA, Patel R, et al. The development of acute lung injury is associated with worse neurologic outcome in patients with severe traumatic brain injury. J Trauma. 2003:55:106-11.

36. Zambon M, Vincent JL. Mortality rates for patients with acute lung injury/ ARDS have decreased over time. Chest. 2008;133:1120-7.

37. Shahrokhi N, Khaksari M, Soltani Z, Mahmoodi M, Nakhaee N. Effect of sex steroid hormones on brain edema, intracranial pressure, and neurologic outcomes after traumatic brain injury. Can J Physiol Pharmacol. 2010;88:414-21.

38. Ley EJ, Short SS, Liou DZ, Singer MB, Mirocha J, Melo N, et al. Gender impacts mortality after traumatic brain injury in teenagers. J Trauma Acute Care Surg. 2013;75:682-6.

39. Albrecht JS, McCunn M, Stein DM, Simoni-Wastila L, Smith GS. Sex differences in mortality following isolated traumatic brain injury among older adults. J Trauma Acute Care Surg. 2016;81:486-92.

40. Tanaka T, Imai T. Pubertal maturational in health girls and standardization ofmenarche age. J Jpn Pediatr Soc. 2005;109:1232-42.

41. Skolnick BE, Maas Al, Narayan RK, van der Hoop RG, MacAllister T, Ward $J \mathrm{D}$, et al. A clinical trial of progesterone for severe traumatic brain injury. $\mathrm{N}$ Engl J Med. 2014;371:2467-76.

42. Wright DW, Yeatts SD, Silbergleit R, Palesch YY, Hertzberg VS, Frankel M, et al. Very early administration of progesterone for acute traumatic brain injury. N Engl J Med. 2014;371:2457-66.

43. Wagner AK, Bayir H, Ren D, Puccio A, Zafonte RD, Kochanek PM. Relationships between cerebrospinal fluid markers of excitotoxicity, ischemia, and oxidative damage after severe TBI: the impact of gender, age, and hypothermia. J Neurotrauma. 2004:21:125-36.

44. Bambach MR, Mitchell RJ, Grzebieta RH, Olivier J. The effectiveness of helmets in bicycle collisions with motor vehicles: a case-control study. Accid Anal Prev. 2013;53:78-88.

45. Roozenbeek B, Lingsma HF, Lecky FE, Lu J, Weir J, Butcher I, et al. Prediction of outcome after moderate and severe traumatic brain injury: external validation of the International Mission on Prognosis and Analysis of Clinical Trials (IMPACT) and Corticoid Randomisation after Significant Head injury (CRASH) prognostic models. Crit Care Med. 2012;40:1609-17.

\section{Publisher's Note}

Springer Nature remains neutral with regard to jurisdictional claims in published maps and institutional affiliations.
Ready to submit your research? Choose BMC and benefit from:

- fast, convenient online submission

- thorough peer review by experienced researchers in your field

- rapid publication on acceptance

- support for research data, including large and complex data types

- gold Open Access which fosters wider collaboration and increased citations

- maximum visibility for your research: over $100 \mathrm{M}$ website views per year

At BMC, research is always in progress.

Learn more biomedcentral.com/submissions 